CLAWAR 2018: 21st International Conference on Climbing and Walking Robots and the Support Technologies for Mobile Machines, Panama City, Panama, 10-12 September 2018

\title{
PROPOSAL OF PROPULSION UNIT BASED ON EARTHWORM SETAE FOR UNDERWATER EXCAVATION ROBOT
}

\author{
NAOAKI TADAMI, KEITA ISAKA, \\ TOYOHARU NAKATAKE, AMI FUJIWARA \\ YASUYUKI YAMADA, TARO NAKAMURA \\ Faculty of Science and Engineering, Chuo University, \\ 1-13-27 Kasuga, Bunkyo-ku, Tokyo, 112-8551, Japan
}

MAKOTO SUGESAWA, HIROSHI YOSHIDA

Japan Agency for Marine-Earth Science and Technology, Yokosuka, Kanagawa, Japan

\begin{abstract}
In this paper, we developed a propulsion unit with bristles imitating the setae of earthworm. This propulsion unit is installed in SEAVO: sub-seafloor excavation robot. To realize underwater excavation by SEAVO, it is necessary to move the soft and fluid sedimentary layer of seafloor surface. As a solution, we focused on the setae of earthworm which live in mud and soft soil. Then, we developed the propulsion unit with bristles imitating the earthworm's setae and measured the performance of the bristlesattached propulsion unit. Comparing the measurement result of the bristles-attached propulsion unit with the previous propulsion unit, we confirmed the usefulness of the bristles-attached propulsion unit.
\end{abstract}

Keywords: Excavation robot, earth auger, Seabed exploration

\section{Introduction}

The ocean occupies about $70 \%$ of the earth's surface area. Recent studies have confirmed the existence of various mineral resources on this vast seabed. ${ }^{1}$ To elucidate the reserves and generation process of these resources, it is necessary to explore the detail of seabed in a wide area. One of the seabed exploration has sampling. ${ }^{2}$ In the current exploration, descending equipment such as drill string from the research vessel to collect soil at the seabed. ${ }^{3}$ However, as the water depth gets deeper, exploration equipment and vessels become larger the size and increase the operation cost. In addition, since the area of these exploration is limited to one spot, it is not suitable for investigation of wide area. This problem can be improved by the cost reduction and the high efficiency exploration. Therefore, we will develop the robot that explore freely under seabed. Then, we realize low-cost and highly effective exploration by multiple robots. 
In the previous research, we developed a flexible earth auger and propulsion unit for bending excavation. ${ }^{4-5}$ We have developed the robot "SEAVO" which can be excavated and bent and confirmed its usefulness in some experiments. ${ }^{6}$

As the next stage of research, we propose excavation experiment underwater by SEAVO. To achieve underwater excavation, it is necessary to propel and excavate underwater ground rich in fluidity. In this paper, we propose the bristles-attached propulsion unit which improves the SEAVO's propulsion ability developed. First, we perform underwater excavation experiment with only the excavation unit and measure the torque generated by excavation. The propulsion unit must support this torque. Next, we develop the bristles-attached propulsion unit and measure the performance of this propulsion unit. Finally, we compare these results of experiments and evaluate whether propulsion unit can support the excavation torque of the target value: $4.79 \mathrm{~N} \mathrm{~m}$.

\section{Concept of SEAVO}

Figure 1 shows the future vision of SEAVO. SEAVO is dropped from the ship to the seabed with the launcher. The launcher is a support rack of a robot and equipped with robot power and communication equipment. The exploration operation by SEAVO is divided into the five stages.

Phase 1. After arriving at the seabed, SEAVO start excavation from the launcher. Phase 2. SEAVO excavates horizontally and performs the wide area exploration. Phase 3. Transporting the seabed's sample, collecting the sample to the launcher. Phase 4. After sampling, SEAVO moves backward and returns to the launcher. Phase 5. The launcher floats up to the sea surface and is collected by vessels.

In this paper, we focus on Phase 1 and discuss the prototype of robot which can excavate in the linear direction underwater.

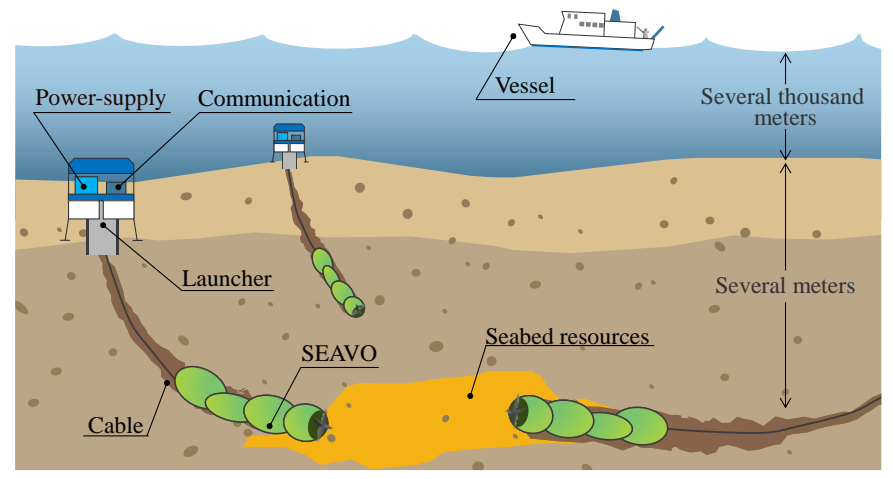

Figure 1. Concept of sub seafloor excavation robot(SEAVO). 
The overview of SEAVO is shown in Fig. 2 (a). This robot consists of an excavation unit and a propulsion unit. The overview of the excavation unit and the propulsion unit are shown in Fig. 2 (b) and Fig. 2 (c). The excavation unit has a role of securing a space for propelling. The propulsion unit has a role of the propulsion operation and the support of excavation reaction force.

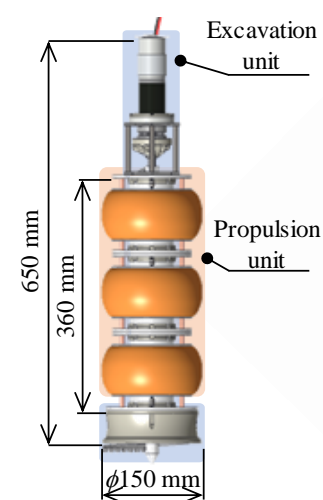

(a) SEA VO

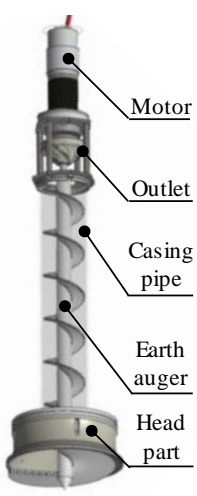

(b) Excavation unit

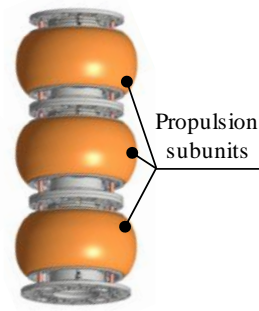

(c) Propulsion unit

Figure 2. Overview of the excavation robot.

\section{Hurdle of underwater excavation}

In previous study, we confirmed the excavation and propulsion operation of SEAVO in land experiments. To realize underwater excavation by SEAVO, we will discuss the hurdle underwater environment and their countermeasures.

There is the problem that conventional propulsion units will not function in underwater. Unlike the onshore test, the ground is fluid, so it is difficult for the propulsion unit to support the excavation. So, when large reaction force of excavation occurs, excavation and propulsion are impossible, such as rotation of the robot body. Therefore, the propulsion unit functions reliably in fluid ground is important. About this problem, we focus on important excavation torque among the excavation reaction force and discuss it. Therefore, experimentally improve the gripping torque by biomimetic utilizing the characteristics of organisms which living underground.

Several ideas can be cited as an improvement plan for the gripping torque of the propulsion unit that supports the excavation torque. However, depending on the countermeasure, the propulsion movement may be affected. Also, it was difficult to analyze like the simulation in underwater environment. Therefore, we try to improve gripping torque by using the characteristics of living things in underground. 


\subsection{Setae of Earthworm}

As a method of improving gripping torque, we focused on the characteristic of earthworm. The structure of the earthworm is shown in Fig. 3. On the body surface of earthworms, hard bristles called setae are existing. Earthworm can fix soil like this anchor with these setae. As a result, earthworms can move in soft mud. We install the mechanism imitating this setae in the propulsion unit and improve the gripping torque without hindering the propulsion movement.

\subsection{Setae attached propulsion unit}

In this section, we explain the setae attached propulsion unit. Fig. 4 shows the appearance and gripping of setae attached propulsion unit. Like the setae of earthworm, when the propulsion unit contracts, there is little influence on the surroundings of the unit, and at the time of expansion, the setae widen, the frictional force increases, and the gripping torque improves. For the evaluation of the propulsion unit, it is necessary to decide the excavation torque generated by underwater excavation. However, the behavior of the soil in the underwater environment is very complicated, so it is extremely difficult to elucidate theoretically. Therefore, experimentally underwater excavation experiment of the excavation unit is performed, and the excavation torque is obtained. Then, we confirm from the comparison of experimental results whether the proposed propulsion unit can support excavation of SEAVO.

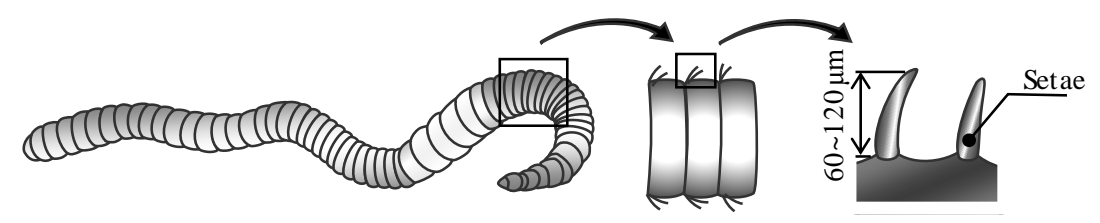

Figure 3. Setae of earthworm.

(a)

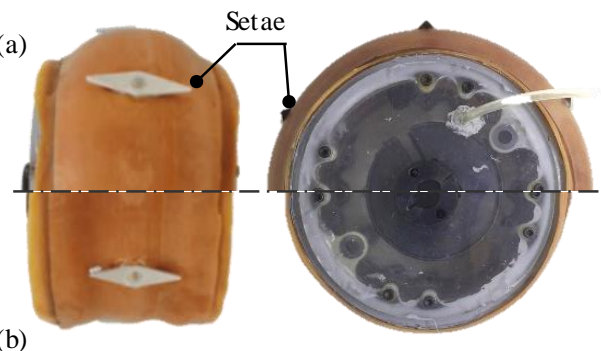

(c)

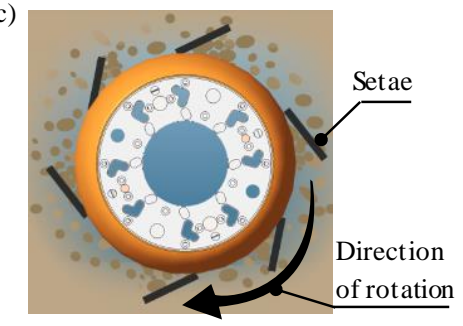

Figure 4. Appearance and gripping of setae attached propulsion unit. a) Pressurized propulsion subunit at $0.03 \mathrm{MPa}$. b) Unpressurized propulsion subunit. c) Gripping of propulsion subunit with setae. 


\section{Underwater excavation of the excavation unit}

In this section, we carry out underwater excavation experiment of an excavation unit to measure excavation torque during excavation. Then, the excavation torque measured by the experiment is set as the target gripping torque of the propulsion unit.

\subsection{Experiment setup of excavation torque}

The experiment equipment of excavation unit is shown in Fig. 5. This equipment consists of excavation unit, slide rail, aluminum frame, and simulated sedimentary soil tank. In addition, Toyoura Silicone Sand (Toyoura Silicone Industry) was used as simulated sediment. Various things such as sand, mud and plankton carcasses are sediment on seafloor. However, reproducing this is difficult due to the diversity and non-uniformity of the marine sediment. Therefore, we experimentally selected silica sand which is one main component of the marine sediment and whose granularity is uniform from the viewpoint of reproducibility of the experiment.

The excavation unit is fixed to the aluminum frame so as not to rotate, and lows in the vertical direction by the slide rail. In the experiment. Also, the earth auger is rotated at $10 \mathrm{rpm}$, the pressing force is the mass of the excavating unit, and the mass of the excavating unit is $2.1 \mathrm{~kg}$. Then, the excavation torque is calculated from the current flowing in the motor (RS - 775 GM 504, Suzaku Giken).
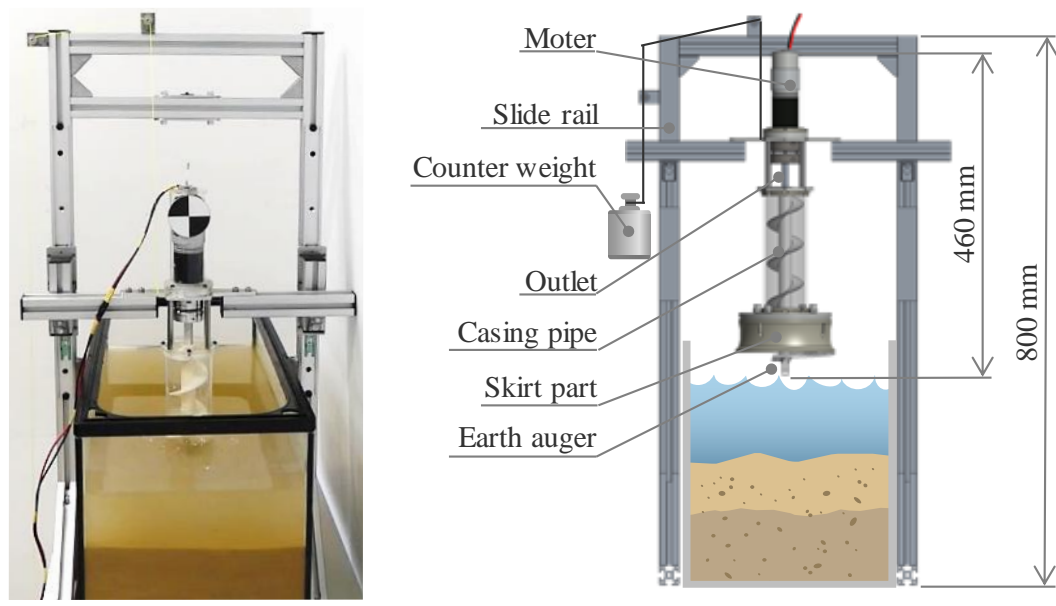

Figure 5. Experiment setup of excavation unit. 


\subsection{Experimental result and discuss}

The excavation process and the result of the excavation torque are shown in Fig. 6. After $67 \mathrm{~s}$, excavation unit discharged soil from the outlet part, and it reached the lowest end of the movable range, we ended the experiment in $186 \mathrm{~s}$. Next, the results of the torque are shown in Fig. 6. From this figure, the maximum excavation torque was $9.58 \mathrm{~N} \mathrm{~m}$ at $85 \mathrm{~s}$. Because the propulsion unit always holds with two subunits, it needs gripping torque than $4.79 \mathrm{~N} \mathrm{~m}$ per one subunit. Therefore, the target gripping torque is set to $4.79 \mathrm{~N} \mathrm{~m}$.

In addition, focusing on the result of the torque in Fig. 6, Torque is rising from around $50 \mathrm{~s}$. we considered the influence of soil density as a factor that the Increase in torque. The surface layer of the sedimentary soil contains a lot of water, and it is soft. Because of that, we considered that the soil entry speed of the unit is faster, and a large torque has occurred. On the other hand, the soil became hard with the depth, and soil entry velocity declined by earth pressure and friction. As a result, the excavation time became longer, and the torque decreased. From these facts, we think that there is a relationship between excavation speed and torque.

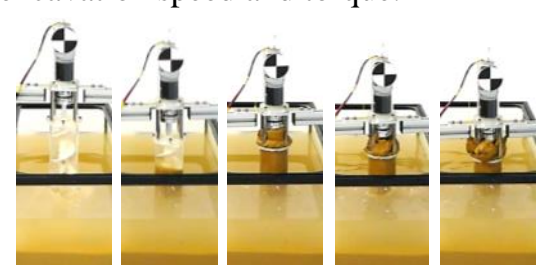

(a) $0 \mathrm{~s}$

(b) $36 \mathrm{~s}$

(c) $72 \mathrm{~s}$ (d) $108 \mathrm{~s}$ (e) $144 \mathrm{~s}$

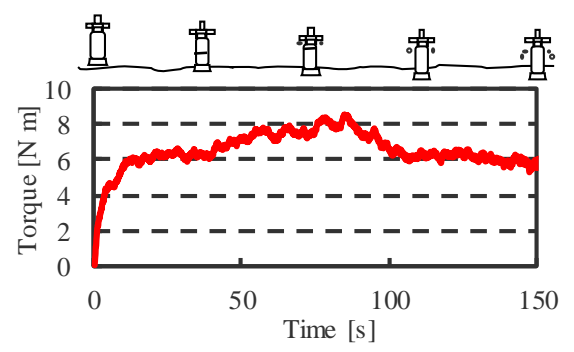

Figure 6. Excavation process and result of excavation torque.

\section{Experiment of propulsion unit}

In this section, to confirm the usefulness of the setae attached propulsion unit proposed in section III, gripping torque is measured and compared with conventional propulsion unit.

\subsection{Experiment setup of gripping torque}

The experiment equipment is shown in Fig. 7. This equipment consists of propulsion subunit, aluminum frame, and simulated sedimentary soil tank. In the experiment, the propulsion subunit was buried in the sedimentary soil tank, and the pressure of the subunit applied to hold the inner wall. Then, a force was 
applied in the rotating direction to measure the magnitude of the force at the time of movement, using a force gauge, and the grip torque was calculated.
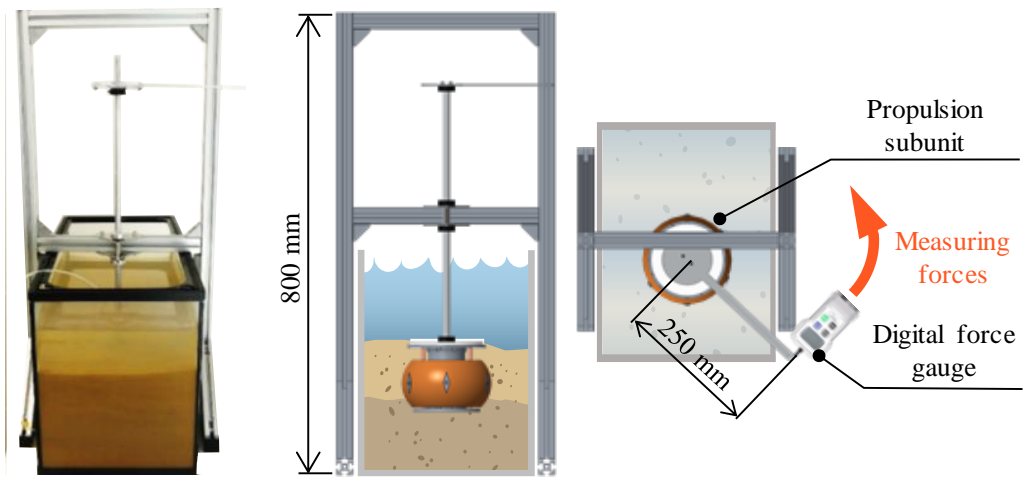

Figure 7. Experiment setup of propulsion unit.

\subsection{Experimental result and discuss}

Measurement environment and the result of gripping torque are shown in Fig. 8. From the experimental result, it was confirmed that the gripping torque of the propulsion subunit can be improved by attaching the setae. The maximum gripping torque of the conventional propulsion subunit was $3.5 \mathrm{~N} \mathrm{~m}$ when 30 $\mathrm{kPa}$ was applied. Therefore, it was insufficient for the $4.79 \mathrm{~N} \mathrm{~m}$ required to support excavation. In the experimental result, the maximum gripping torque of the propulsion subunit with the six setae attached was $5.8 \mathrm{~N} \mathrm{~m}$ for $30 \mathrm{kPa}$. From this result, it was confirmed that the propulsion subunit with setae-attached achieved the target value of $4.79 \mathrm{~N}$ m by applying $30 \mathrm{kPa}$.
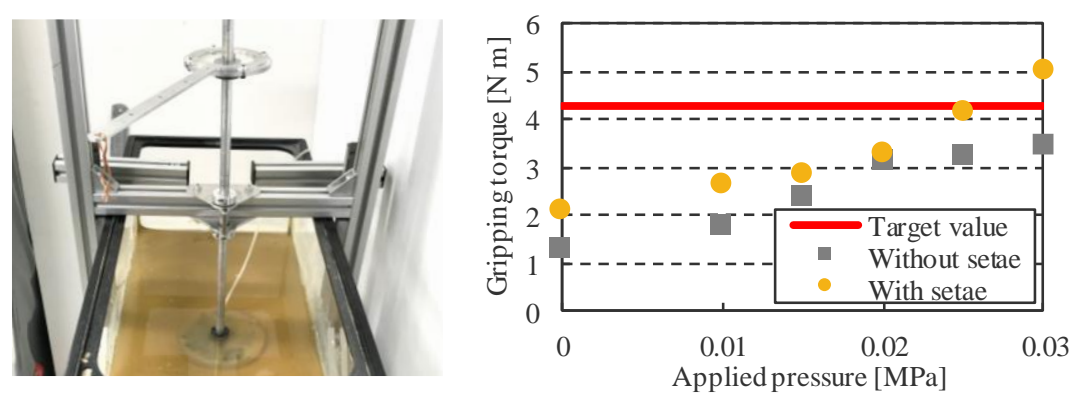

Figure 8. Measurement environment and result of gripping torque. 


\section{Conclusion}

In this paper, we presented a setae-attached propulsion subunit based on earthworm setae that realizes underwater excavation. The conclusions reached in this paper are presented below: Setae attached propulsion subunit inspired by the earthworm setae was presented. The following future work is being considered.

- Setae attached propulsion subunit inspired by the earthworm setae was presented.

- The performance of the setae-attached propulsion subunit was confirmed via characteristic experiments.

- Experimental results confirmed that the setae-attached propulsion unit exceeds the target grip torque and showed usability.

\section{Future works}

The following future work is being considered.

- We will examine a new underwater excavation unit so that the unit can excavate a lower torque.

- We will confirm whether we can excavate excavation robot underwater equipped with the new propulsion unit proposed.

\section{Reference}

1. S. Scott, "Marine Minerals: Their Occurrences, Exploration and Exploitation", Oceans2011, IEEE, pp. 1-8, 2011.

2. H. Yoshida, T. Aoki, H. Osawa, S. Ishibashi, Y. Watanabe, J. Tahara, T. Miyazaki and K. Itoh, "A Deepest Depth ROV for Sediment Sampling and Its Sea Trial Result", Underwater Technology and Workshop on Scientific Use of Submarine Cables and Related Technologies, 2007.

3. Wada. K, "Coring Technology to be applied in IODP NAnTroSEIZE," OCEANS2008-MTS/IEEE Kobe Techno-Oceans, 1 (2008).

4. M. Nagai, C. Hirabayashi, Y. Yamada, T. Nakamura, and H. Yoshida, "Development of a flexible excavation unit for a peristaltic crawling seabed excavation robot," Proc. Int. Conf. on CLAWAR, pp.4950-4955, 2016.

5. N. Tadami, A. Fujiwara, T. Nakatake, M. Nagai, Y. Yamada, T. Nakamura, H.Yoshida, H. Sawada, and T.Kubota, "DEVELOPMENT OF A FLEXIBLE PROPULSION UNIT FOR A SEABED EXCAVATION ROBOT" Proc. Int. Conf. on CLAWAR, pp.4950-4955, 2017.

6. N. Tadami, M. Nagai, T. Nakatake, A. Fujiwara, Y. Yamada, T. Nakamura, H. Yoshida, H. Sawada and T. Kubota, "Curved Excavation by a Subsafloor Excavation Robot”, Proc. Int Conf on IROS, pp.4950-4955, 2017. 